\title{
Multiple Scale Modeling of Geophysical Processes
}

\author{
Eugénio M. Rocha ${ }^{1}$ - Francisco Curado ${ }^{2}$
}

Published online: 4 June 2018

(C) International Association for Mathematical Geosciences 2018

This special issue of Mathematical Geosciences is the outcome of the Mathematics and Engineering in Marine and Earth Problems - MEME'2014 international conference, which was held 21-25 July 2014 in Aveiro, Portugal. This conference was sponsored by the University of Aveiro and organized by Eugénio M. Rocha and Francisco Curado, in the context of the international project "Mathematics of Planet Earth 2013", which continued in 2014 as "Mathematics of Planet Earth" (MPE). The event was organized with the objective of creating a multidisciplinary forum where mathematicians, engineers, and other scientists could meet to identify fundamental research questions and jointly engage in the development of innovative mathematical and numerical tools for the study and exploration of the earth and oceans. The conference included 49 presentations by participants from 14 countries, distributed by eight thematic sessions, and featured nine plenary talks by an international panel of leading keynote speakers. The topics discussed included: models of pollutant transport in compacted materials, optimal control for environmentally sustained management of resources in agriculture, modelling and control of wildfires, ice flow modelling of glaciers and ice sheets, inversion of seismic oceanography data, prediction of oil reservoir performance, ocean exploration by networked robotic systems and sensors, and modelling of

$凶$ Francisco Curado

fcurado@ua.pt

Eugénio M. Rocha

eugenio@ua.pt

1 Center of Research and Development in Mathematics and Applications (CIDMA)/Department of Mathematics (DMAT), University of Aveiro, Aveiro, Portugal

2 Institute of Electronics and Informatics Engineering of Aveiro (IEETA)/Department of Electronics and Informatics Engineering (DETI), University of Aveiro, Aveiro, Portugal 
atmospheric convection and extra-tropical storms, and optimal control interventions in tuberculosis-HIV/AIDS. The full conference program, including the complete list of speakers, the book of abstracts, and the collection of invited and contributed talks, can be found at www.meme.glocos.org.

This special issue comprises five papers, constituting a good illustration of the work presented at the conference, which was motivated by a number of realistic, geophysical-related problems of environmental, economic, and societal interest. Because of space limitations, some of the remaining papers have been published in normal issues. The common thread of these contributions is the presentation and discussion of mathematical models of geophysical processes, supported by rigorous theoretical developments and relying on real data and realistic numerical experiments for their validation or benchmarking.

The containment of high-level radioactive waste in geological formations and the isolation of household disposal sites using expansive compacted bentonites motivates the contributed paper of Fatiha Bouchelaghem titled, "Multi-scale Study of Pollutant Transport and Uptake in Compacted Bentonite". Borrowing from prior work of the author on simulated tests of lead nitrate percolation, the present essay deals with the modelling of pollutant transport and pollutant fixation on the smectite layers' surface. The results presented in the study tend to evidence that some usual assumptions relative to the transport of solute in compacted water-saturated bentonites may not be valid under moderate compaction states and important structural variations. A model of contaminant uptake and transport is proposed based on the homogenization method for locally periodic structures. The results of finite element calculations of the effective diffusion tensors at the mesoscopic and macroscopic scales are compared with available measurements in order to assess the underlying model assumptions, as well as the representability of the idealized microstructures employed in the calculations.

The paper of Ana González-Nicolás et al. entitled "Application of Binary Permeability Fields for the Study of $\mathrm{CO}_{2}$ Leakage from Geological Carbon Storage in Saline Aquifers of the Michigan Basin", addresses the hot topic of geological carbon storage (GCS), a promising approach to reduce $\mathrm{CO}_{2}$ emissions from power plants into the atmosphere. Deep saline aquifers are considered ideal formations for GCS due to their larger storage capacity compared to other targets such as depleted gas/oil reservoirs, but present the disadvantage of having limited characterization data, specifically about the continuity of the overlying sealing formations. The present essay advances a methodology for preliminary uncertainty quantification of $\mathrm{CO}_{2}$ leakage at sites of GCS with little or no information on the sealing properties. The work includes the study of a candidate GCS site located in the Michigan Basin, whose sealing properties of the caprock are practically unknown. The implemented analysis highlights the importance of being able to characterize caprock sealing properties over large areas such as the one considered in the case study.

The work entitled "Simulating Oxygen Intrusion into Highly Heterogeneous Fractured Media Using High Performance Computing" of Paolo Trinchero et al. discusses a number of sensible issues related with the use of crystalline rocks as host formations for high-level nuclear waste repositories. In particular, the authors discuss modelling and numerical simulation issues that affect the study of the redox evolution of these host rock formations and its impact on the stability and safety of the disposal sites. 
The authors advert to the limitations of basic geometrical models and simplified representations of the physical/mineralogical heterogeneity of the sites, which are often employed in the evaluation of oxygen penetration in rock formations. They further propose and demonstrate the efficiency of a novel numerical framework combined with high performance computing methods, which is designed to carry out site-scale reactive transport calculations of oxygen intrusion in fractured media, including the full geometrical, physical, and mineralogical complexity of the site.

Ocean bottom seismometers (OBS) are self-contained data-acquisition systems placed on the ocean floor, which record seismic data generated by airguns and earthquakes. They constitute fundamental tools of geophysical investigations including the study of Earth's deep crustal structure and oil and gas exploration. OBS deployment is normally performed by free fall from the deck of oceanographic vessels to the ocean floor, a procedure that leads to large uncertainty of positioning of the OBS nodes. Accurate seismometer positioning, which constitutes a key step in the processing flow of the acquired data, is a challenging nonlinear problem whose complexity is increased by the need to solve for the varying velocity of sound propagation in the water layer.

In their paper titled "Accurate Ocean Bottom Seismometer Positioning Method Inspired by Multilateration Technique", Omar Benazzouz et al. propose a linearization of the OBS positioning problem via a multilateration technique that solves jointly for the average water layer velocity and the OBS position using only shot locations and first arrival times as input data. An accuracy assessment of the method is presented using a large control dataset acquired onboard a research vessel of the U.S. Geological Survey in the Gulf of Mexico in 2013.

The paper of Carlos Marques and José Castanheira titled "Diagnosis of Free and Convectively Coupled Equatorial Waves", discusses the performance of current models of atmospheric convection in the tropical region, a complex process that plays an important role in the global climate. They present a methodology for diagnosis of free and convectively coupled equatorial waves, which is illustrated for Kelvin and mixed Rossby-gravity waves. The method proposed is applied to real and synthetic data-sets, which include: geopotential and horizontal wind fields produced by the European Centre for Medium-Range Weather Forecasts global atmospheric reanalysis, outgoing longwave radiation data produced by the National Oceanic and Atmospheric Administration, and data simulated by two climate models that participated in the fifth phase of the Climate Model Intercomparison Project. The methodology is proposed as a useful procedure for climate model validation and benchmarking that could help the identification of which physical processes need to be improved in current climate models.

The guest editors acknowledge the contributions and the patience of the authors, as well as the work of all the participants in the MEME'2014. They also wish to acknowledge with thanks the kind availability and the careful work of the reviewers. 